Conclusions Our small study shows that IABP support does not result in reduced in-hospital mortality in patients presenting with cardiogenic shock. Morality remains high despite advance in medical treatment.

Conflict of Interest Cardiology

\section{NICE CHALLENGE THAT IS DIFFICULT TO MEET?}

${ }^{1}$ Vasanth Sritharan, ${ }^{1}$ Mohammed Sallam, ${ }^{1}$ Mohammad Islam, ${ }^{2}$ Atif Siddiqui, ${ }^{1}$ Ganesan Kumar. 'Luton and Dunstable University Hospital, Luton, UK; ${ }^{2}$ University College London

\subsection{6/heartjnl-2021-BCS.75}

Introduction Current NICE guidelines recommend early invasive strategy with coronary angiography within 72 hours of first presentation of NSTEMI to those who have an intermediate or higher risk of adverse cardiovascular events, defined as a GRACE 2.0 risk greater than 3.0\%. The aim of this audit is to explore NICE guideline adherence, patient characteristics and clinical outcomes.

Methods Retrospective data from patients with a final diagnosis of NSTEMI in the months of October 2018 and January 2019 was gathered from clinical coding. Patients were stratified by their GRACE 2.0 score, which was retrospectively calculated using their clinical notes and investigations on the Integrated Clinical Environment.

Results There were 116 patients of which 90 (77.6\%) had an intermediate to high risk. Data was analysed for all the 90 patients (table 1) with further analysis for those who underwent early or late inpatient coronary angiography with fisher's exact test (table 2).

From the 90 patients $44(48.9 \%)$ underwent coronary angiography, while 46 patients (51.1\%) were not suitable for invasive intervention hence treated with standard medical therapy. 18 patients (40.9\%) had coronary angiography within 72 hours and 26 waited more than 72 hours. 13 (72.2\%) of those who had early coronary angiography went on to have

Abstract 75 Table 1 Summary table of patients with intermediate to high risk of cardiovascular event

\begin{tabular}{llll}
\hline $\begin{array}{l}\text { Clinical characteristic } \\
(\mathrm{N}=90)\end{array}$ & $\begin{array}{l}\text { Coronary } \\
\text { angiography } \\
>72 \mathrm{hrs}(\mathrm{N}=18)\end{array}$ & $\begin{array}{l}\text { Coronary } \\
\text { angiography } \\
>72 \mathrm{hrs}(\mathrm{N}=26)\end{array}$ & $\begin{array}{l}\text { Medical } \\
\text { management } \\
(\mathrm{N}=46)\end{array}$ \\
\hline $\begin{array}{l}\text { Demographic } \\
\text { Age (mean) }\end{array}$ & 70.9 & 74.7 & 82.1 \\
$\begin{array}{l}\text { Female } \\
\text { Secondary prevention }\end{array}$ & $8(44.4 \%)$ & $10(38.5 \%)$ & $27(58.7 \%)$ \\
$\begin{array}{l}\text { DAPT or antiplatelet } \\
+ \text { anticoagulation }\end{array}$ & $17(94.4 \%)$ & $24(92.3 \%)$ & $37(80.4 \%)$ \\
ACEi & $17(94.4 \%)$ & $23(88.5 \%)$ & $27(58.7 \%)$ \\
Statin & $18(100 \%)$ & $25(96.2 \%)$ & $33(71.7 \%)$ \\
Intervention & & $10(38.5 \%)$ & $0(0 \%)$ \\
PCI & $13(72.2 \%)$ & $3(11.5 \%)$ & $0(0 \%)$ \\
CABG & $0(0 \%)$ & & $13(21.7 \%)$ \\
Outcome & & $10(21.7 \%)$ \\
Dead at 6 months & $0(0 \%)$ & $7(26.9 \%)$ & \\
Re-admitted with & $5(27.8 \%)$ & & \\
adverse cardiac event at & & & \\
1 year & & & \\
\hline
\end{tabular}

Abstract 75 Table 2 Comparison of adverse cardiovascular events when coronary angiography is delayed

\begin{tabular}{|c|c|c|c|}
\hline & $\begin{array}{l}\text { Coronary } \\
\text { angiography }>72 \mathrm{hrs} \\
(\mathrm{N}=18)\end{array}$ & $\begin{array}{l}\text { Coronary } \\
\text { angiography }>72 \mathrm{hrs} \\
(\mathrm{N}=26)\end{array}$ & $\begin{array}{l}\mathrm{p} \\
\text { value }\end{array}$ \\
\hline Death at 6 months & $0(0 \%)$ & $1(3.8 \%)$ & 1.0000 \\
\hline $\begin{array}{l}\text { Re-admitted with adverse } \\
\text { cardiac event at } 1 \text { year }\end{array}$ & $5(27.8 \%)$ & $7(26.9 \%)$ & 1.0000 \\
\hline
\end{tabular}

percutaneous coronary intervention compared to the $10(38.5 \%)$ patients who had delayed coronary angiography $(\mathrm{p}=0.0018)$. Conclusion Despite expectations there was only $40.9 \%$ adherence to NICE guideline. In contrast to expectations intermediate to high-risk patients are less likely to have early invasive strategy. However, there is no difference in outcome between an early versus late invasive strategy for inpatients. Standard medical treatment is still practiced at higher number of patients with multiple comorbidities, higher age and intermediate to high risk. This shows that meeting NICE recommendations can be challenging for numerous factors beyond a clinician's control. Further data from medium sized district general hospitals with catheterization laboratory facility is needed to assess the overall ability of these centres to meet NICE recommendations.

Conflict of Interest None

\section{EVOLUTION OF THE CORONARY ARTERY STENT - 35 YEARS ON (POSTER)}

Joshua Welsh, George Choa, Shagana Karunainathan, Arnav Srivastava, Dhivyaa Premachandra, Sadat Edroos. Luton \& Dunstable University Hospital, Bedfordshire Hospitals NHS Foundation Trust, Luton, UK

\subsection{6/heartjnl-2021-BCS.76}

Coronary artery stents have created the revolutionary field of interventional cardiology. Since their conception in 1964, to the first coronary angioplasty performed in 1977, multidisciplinary work has made what seemed like incredible and outlandish minimally invasive reopening of coronary occlusion into a commonplace mainstay of treatment, reducing mortality from cardiovascular disease globally.

Since the first stent implantation in 1986 the past 35 years of research have seen iterative progress. We have witnessed ever-evolving stent design. Drug-eluting stents resulted in a reduction in post-implantation restenosis and thrombosis previously seen with bare metal stents. Stent platforms have offered improved deliverability, circumferential strength and thinner stent struts, such that we are now able to offer tailored stent delivery to the most tortuous and bifurcated of vessels, in territories that were once seen as insurmountable. Bioresorbable stents also promised a further revolution, with hopes of complete elution of drug, resorption of the delivery mechanism, and reduced inflammatory response, but ultimately underwhelming and inferior results. As drug eluting balloons offer an alternative goal of restoring lumen patency through dilatation and targeted drug delivery, in the absence of the physical scaffold, we review the progress of percutaneous coronary intervention over the lifetime of our speciality and look 